\title{
Soluble TWEAK independently predicts atherosclerosis in renal transplant patients
}

\author{
Kultigin Turkmen ${ }^{1 *}$, Halil Zeki Tonbul ${ }^{2}$, Fatih Mehmet Erdur ${ }^{2}$, Aysun Toker ${ }^{4}$, Zeynep Biyik², Huseyin Ozbiner ${ }^{3}$, \\ Abduzhappar Gaipov², Elvin Enes Gull, Mehmet Kayrak ${ }^{5}$, Yalcin Solak², Orhan Ozbek ${ }^{3}$, Suleyman Turk² \\ and Adrian Covic ${ }^{6}$
}

\begin{abstract}
Background: Cardiovascular risk is increased in the early stages of chronic kidney disease (CKD) and also found to be ongoing in renal transplant (Rtx) patients. As a sign of atherosclerosis, increased carotid intima-media thickness (CIMT) has been widely accepted as a strong predictor of cardiovascular disease (CVD) and mortality in CKD patients. A novel markers, soluble tumor necrosis factor-like weak inducer of apoptosis (STWEAK) and neutrophil-to-lymphocyte ratio (NLR) were introduced as potential markers in inflammatory disorders including CKD. The role of Rtx in terms of atherogenesis is still unclear. We aimed to investigate the relationship between sTWEAK, NLR and CIMT in Rtx patients without overt CVD and to compare these results with those obtained from healthy subjects.

Methods: Cross-sectional analysis in which CIMT measurements, NLR and serum TWEAK levels were assessed in 70 Rtx patients ( 29 females; mean age, $40.6 \pm 12.4$ years) and 25 healthy subjects (13 females, mean age; $37.4 \pm 8.8$ years).

Results: STWEAK levels were significantly decreased $(p=0.01)$ and hs-CRP, NLR and CIMT levels of Rtx patients were significantly increased compared to healthy subjects $(p<0.0001, p=0.001, p<0.0001$, respectively). sTWEAK was also found to be decreased when eGFR was decreased ( $p=0.04$ between all groups). CIMT was positively correlated with sTWEAK and NLR in Rtx patients $(r=0.81, p<0.0001$ and $r=0.33, p=0.006$, respectively). sTWEAK was also positively correlated with NLR ( $r=0.37, p=0.002)$. In the multivariate analysis only sTWEAK was found to be an independent variable of increased CIMT.
\end{abstract}

Conclusion: STWEAK might have a role in the pathogenesis of ongoing atherosclerosis in Rtx patients.

Keywords: STWEAK, Neutrophil-to-lymphocyte ratio, Carotid intima-media thickness, Renal transplantation

\section{Background}

Despite improvements in diagnostic tools and therapeutic approaches, premature death related to cardiovascular disease (CVD) remains the most common cause of death in patients with chronic kidney disease (CKD) [1]. Cardiovascular risk is increased even in the early stages of CKD and this heightened risk is also found after renal transplantation (Rtx) [2,3]. Besides traditional risk factors including hypertension, diabetes, dyslipidemia, advanced age and left ventricular hypertrophy (LVH), novel risk factors such as endothelial dysfunction (ED), vascular calcifications, oxidative stress, and inflammation

\footnotetext{
* Correspondence: mdkt2010@yahoo.com

1 Department of Nephrology, Erzincan University Mengucek Gazi Training and Reseach Hospital, Erzincan, Turkey

Full list of author information is available at the end of the article
}

are highly prevalent and seem to play a more important role in renal patients compared to healthy subjects [4-7]. Several studies suggested that persistent systemic inflammation could be the main factor responsible for the increased risk in ESRD patients, regardless of the renal replacement therapy [8]. To prove this hypothesis, researchers analyzed a large panel of biomarkers to fully characterize the relation between inflammation and CVD, including C-reactive protein, interleukin (IL)-1 $\beta$, IL-6, tumor necrosis factor- $\alpha$ (TNF- $\alpha$ ) [8,9], as well as several interesting new biomarkers.

Neutrophil-to-lymphocyte ratio (NLR) is a potential marker for inflammation in cardiac and non-cardiac disorders [10-12] that was also shown to be a predictor of long term mortality in patients who underwent percutaneous coronary intervention [13]. We recently demonstrated that

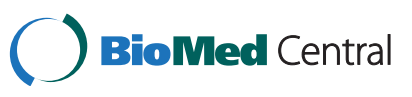


NLR could predict inflammation in ESRD patients [14]. Recently, a novel marker - the soluble tumor necrosis factor-like weak inducer of apoptosis (sTWEAK, TNFSF12) was introduced as a tumor necrosis factor (TNF) related cytokine in various inflammatory and non-inflammatory disorders [15]. To date, a transmembrane protein, fibroblast growth factor-inducible 14 (Fn14) and a scavenger receptor, CD163 were discovered as receptors of sTWEAK $[16,17]$. Binding of sTWEAK to Fn14 mediates multiple effects, including cellular growth, proliferation, migration, differentiation, apoptosis, angiogenesis, fibrogenesis and inflammation [18]. A soluble form of CD163 was also discovered and introduced as a risk factor for long term mortality in patients with peripheral artery disease [19].

Since the association between TWEAK and subclinical atherosclerosis was demonstrated in general and CKD populations $[20,21]$, we aimed to investigate the relationship between sTWEAK, NLR and carotid IMT (CIMT) in Rtx patients without overt CVD and to compare these results with those obtained from healthy subjects.

\section{Methods}

The study protocol was approved by the Medical Ethics Committee of Selcuk University (Meram School of Medicine, Konya, Turkey). Written informed consent was obtained from all subjects included in the study.

This was a cross-sectional study involving 70 Rtx patients (29 females, 41 males; mean age, $40.6 \pm 12.4$ years) followed for at least 6 months in the transplantation unit of the Necmettin Erbakan University and 25 healthy subjects (13 females; mean age, $37.4 \pm 8.8$ years) between January and November 2011.

Patients aged 18-70 years willing to participate in the assessment of CIMT by carotid duplex ultrasonography were screened. A review of medical records (including information on age; sex; weight; medications; primary disease of ESRD) was undertaken. Exclusion criteria were: (i) angina pectoris and/or documented coronary artery disease, (ii) congestive heart failure; (iii) active infection; (iv) autoimmune disease; (v) severe secondary hyperparathyroidism (patients with iPTH > $500 \mathrm{pg} / \mathrm{mL}$ ); (vi) nephrotic-range proteinuria and vii) patients with HIV, HBV and HCV history. Eighty-six RTx patients were screened and 16 patients were excluded from the study. Of the 16 excluded patients - 7 had documented coronary artery disease, 4 congestive heart failure (New York Heart Association (NYHA) class III-IV); 2 active infection; 2 secondary hyperparathyroidism; and 1 patient had autoimmune disease. None of the patients included in the study had nephrotic-range proteinuria and arrhythmia based on electrocardiography (ECG).

Twenty-five age and sex-matched normotensive healthy individuals (13 females, mean age; $37.4 \pm 8.8$ years) referred from outpatient clinics of the Internal Medicine
Department of Necmettin Erbakan University were enrolled as control subjects. They were subjected to the same inclusion and exclusion criteria as the transplanted patients.

Blood pressure was measured in the upright sitting position after $\geq 5$ min of rest using an Erka sphygmomanometer (PMS Instruments Limited, Berkshire, UK) with an appropriate cuff size. Two readings were recorded for each individual. The mean value of the two readings was defined as the blood pressure. Patients with SBP and DBP $\geq 140 \mathrm{mmHg}$ and $\geq 90 \mathrm{mmHg}$ respectively were assumed to be hypertensive. Forty-two patients were taking anti-hypertensive drugs (all patients were on verapamil). Eleven patients were taking an oral calcium-vitamin D combination, and 9 patients were on active vitamin D. Ten patients $(14.3 \%)$ were on intensive insulin and 16 $(22.9 \%)$ were on oral anti-diabetic medication. All 70 Rtx patients, were on prednisolone and mycophenolate mofetil (MMF), 60 (85.7\%) were on tacrolimus, 4 (5.7\%) were on cyclosporine-A (Сyc-A), 2 were on sirolimus $(2.9 \%)$ and $4(5.7 \%)$ were on everolimus therapy. The donor source was living-related in 32 (45.7\%), livingunrelated in 4 (5.7\%) and deceased-donor in 34 (48.6\%) patients. None of the patients had transplant nephrectomy.

\section{Biochemical analyses}

Venous blood samples were drawn after an overnight fast and stored at $-80^{\circ} \mathrm{C}$ for biochemical analyses. Serum creatinine, urea, aspartate aminotransferase (AST), alanine aminotransferase (ALT), calcium, albumin, uric acid, total cholesterol (TC), high density lipoprotein cholesterol (HDL-C), triglyceride (TG) and phosphorus (P) were determined using a Synchron LX20 system (Beckman Coulter, USA) with original Beckman reagents. HDL-C levels were determined by a direct enzymatic method, without precipitation. LDL-C levels were calculated using the Friedewald formula [22]. Serum hs-CRP levels were measured with a high sensitive immunoturbidimetric assay (Diasis Diagnostic System) using an automated clinical chemistry analyzer. Normal range reference interval of hsCRP for adults was accepted as $<10 \mathrm{mg} / \mathrm{L}$.

\section{STWEAK measurements}

Serum TWEAK levels were measured, in both patients and healthy controls, by a commercially available, kit based on enzyme-linked immunosorbent assay (eBiosience, Human TWEAK Instant Elisa, Cat no: BMS2006INST). The results were expressed as $\mathrm{pg} / \mathrm{mL}$. The calculated overall intra-assay coefficient of variation (CV) was $7.9 \%$.

\section{Definition of NLR}

Complete blood counts with automated differential counts, which included total white blood cells, neutrophils and lymphocytes, obtained at the time of admission. 
NLR was calculated as the ratio between neutrophils and lymphocytes, obtained from the same automated blood sample.

\section{Glomerular filtration rate (GFR) assesment}

GFR was calculated according to the simplified version of the Modification of Diet in Renal Disease (MDRD) Study prediction equation formula, GFR $=186$ * Creatin-

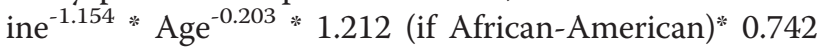
(if female), as defined by Levey [23].

\section{Carotid intima-media thickness measurements}

The carotid intima media thickness recordings were performed by a single investigator who was blinded for patient' charateristics. The carotid arteries were evaluated with the Vivid 7 echocardiography device (General Electrics, Horten, Norway) by using a $10-\mathrm{MHz}$ linear probe. The acquired images were recorded for playback analysis and were later measured off-line. The common carotid artery, the carotid bulb, and internal and external carotid arteries were visualized on both sides. The IMT of the carotid arteries were measured in the distal common carotid artery at a level 15 to $20 \mathrm{~mm}$ proximal to the carotid bulb. The two bright echogenic lines in the arterial wall were identified as the intima and the media. Three measurements were made for each side of the body; separate means were calculated and recorded as the right and left IMT. The intraobserver coefficient of variation for carotid IMT was $2.0 \%$.

Abnormal CIMT was defined as CIMT $\geq 0.82 \mathrm{~mm}$ to characterize atherosclerosis [24].

\section{Statistical analyses}

Statistical analyses were carried out using the Statistical Package for Social Sciences for Windows version 15.0 (SPSS, Chicago, IL, USA). Data are expressed as the mean $\pm \mathrm{SD}$, with a significance level of $\mathrm{P}<0.05$. The normal distribution of all variables was tested using the Kolmogorov-Smirnov Test. Dichotomous variables were compared using the chi-square test. Statistical differences between parametric data of two groups were analyzed using the Student's $t$-test. The Mann-Whitney $U$ test was used to determine differences between non-parametric data. The non-parametric Spearman coefficient of correlation was used to assess correlations between variables without normal distribution. The Rtx patients were divided into tertiles of eGFR. Differences among tertiles were analyzed by one-way ANOVA.

Multivariate linear regression analyses were undertaken to determine independent associations among CIMT and other variables. Age, serum phosphorus, LDL-cholesterol, hs-CRP, NLR, and sTWEAK were entered into the regression model as independent variables, and CIMT was entered as a dependent variable. The backward elimination method was preferred in the stepwise regression analysis and $\mathrm{p}>0.1$ used as a criterion for elimination in this model. At the end of the fifth step, only sTWEAK was remained statistically significant in the model. $\mathrm{p}<0.05$ was considered significant for all tests.

\section{Results}

\section{Patients' baseline characteristics}

The baseline characteristics of the 70 Rtx patients and 25 healthy subjects are depicted in Table 1 . The etiology of renal disease in Rtx patients was chronic glomerulonephritis $(n=6,8.6 \%)$, diabetic nephropathy $(n=6,8.6 \%)$, hypertensive nephropathy $(n=13,18.6 \%)$, polycystic kidney disease $(n=2,3.3 \%)$, tubulointerstitial nephritis $(n=4$, $5.7 \%)$, amyloidosis $(1,1.4 \%)$ and unknown etiology $(n=38$, $53.8 \%)$. Of 70 Rtx patients, $42(60 \%)$ had post-transplant hypertension, 47 (67.1\%) had post-transplant dyslipidemia, and $20(28.6 \%)$ had new-onset diabetes after transplantation (NODAT). There were no differences in age, gender, biochemical parameters including serum LDL and HDL cholesterol, AST, ALT and phosphorus between Rtx patients and healthy subjects. Spot urine protein to creatinine ratio (PCR) values of healthy controls and Rtx patients were $0.01 \pm 0.1$ and $0.3 \pm 0.2$, respectively $(\mathrm{p}<0.0001)$. Serum glucose, urea, creatinine, uric acid, triglyceride and calcium were significantly higher in Rtx patients compared to controls (Table 1). Estimated GFR measurements were also found to be significantly lower in Rtx patients than healthy subjects $(61.7 \pm 22.2$ vs $119.2 \pm 32.9$, respectively, $\mathrm{p}<0.0001)$.

\section{STWEAK, NLR, hs-CRP and carotid intima media thickness measurements}

Results of inflammation and atherosclerosis markers including sTWEAK, hs-CRP, NLR and CIMT are shown in Table 2 with univariate correlates of CIMT and sTWEAK in Rtx patients. sTWEAK levels were significantly decreased $(p=0.01)$ and hs-CRP levels were significantly increased $(\mathrm{p}<0.0001)$ in Rtx patients compared to healthy subjects. Neutrophil count and NLR were also significantly higher in Rtx patients compared to healthy controls ( $\mathrm{p}<0.0001, \mathrm{p}=0.001$, respectively). The mean CIMT of Rtx patients and healthy subjects were $0.72 \pm 0.28 \mathrm{~mm}$ and $0.41 \pm 0.07 \mathrm{~mm}$, respectively $(\mathrm{p}<0.0001)$.

In order to analyze the values of sTWEAK, NLR, hs-CRP, and CIMT according to renal function of Rtx patients, we defined a high eGFR group (group 1) with levels above the 66th percentile (upper tertile) and a low eGFR group (group 3) with levels below the 33th percentile (lower tertile) (Table 3). NLR and CIMT were highest and sTWEAK was lowest in eGFR group 3 (Table 3 ). Furthermore, sTWEAK are significantly decreasing with decreasing levels / tertile of eGFR $(\mathrm{p}=0.04$ between all groups). There was also significant 
Table 1 The baseline characteristics, clinic and laboratory features of the healthy subjects and renal transplant patients

\begin{tabular}{|c|c|c|c|}
\hline Parameters & $\begin{array}{l}\text { Healthy subjects ( } n=25) \\
\text { Median (IQR) }\end{array}$ & $\begin{array}{l}\text { Transplant patients }(n=70) \\
\text { Median (IQR) }\end{array}$ & $P$ value \\
\hline Age (years) & $34(29-43)$ & $38(31-47)$ & 0.17 \\
\hline Female & 13 & 29 & 0.48 \\
\hline BMI $\left(\mathrm{kg} / \mathrm{m}^{2}\right)$ & $27(24-29)$ & $24.8(21.4-28.4)$ & 0.02 \\
\hline SBP $(\mathrm{mmHg})$ & $115(110-120)$ & $122(110-137)$ & $<0.0001$ \\
\hline $\mathrm{DBP}(\mathrm{mmHg})$ & $75(60-80)$ & $80(70-90)$ & 0.001 \\
\hline Glucose (mg/dL) & $92(86-105)$ & $92(86-104)$ & 0.04 \\
\hline Urea(mg/dL) & $25(19-34)$ & $32(24-39)$ & 0.002 \\
\hline Creatinine (mg/dL) & $0.7(0.6-0.8)$ & $1.1(0.8-1.4)$ & $<0.0001$ \\
\hline Albumin (g/dL) & $4.4(4.3-4.5)$ & $4.3(4.1-4.5)$ & 0.02 \\
\hline Uric acid (mg/dL) & $3.7(3.5-4.6)$ & $5.5(3.9-7.0)$ & $<0.0001$ \\
\hline AST(IU/L) & $21(17-22)$ & $21(17-26)$ & 0.25 \\
\hline $\operatorname{ALT}(I U / L)$ & $18(15-20)$ & $19(15-25)$ & 0.18 \\
\hline LDL Cholesterol (mg/dL) & $125(105-137)$ & $118(92-134)$ & 0.38 \\
\hline HDL Cholesterol (mg/dL) & $46(38-50)$ & $46(38-53)$ & 0.69 \\
\hline Triglyceride (mg/dL) & $64(88-147)$ & $144(101-181)$ & 0.001 \\
\hline Calcium (mg/dL) & $8.5(8.5-9.4)$ & $9.5(9.1-9.9)$ & 0.0001 \\
\hline Phosphorus (mg/dL) & $3.0(2.7-3.3)$ & $3.0(2.7-3.6)$ & 0.68 \\
\hline eGFR (ml/min) & $107(91-144)$ & $71(53-90)$ & $<0.0001$ \\
\hline Spot urine & $0.08(0.01-0.2)$ & $0.2(0.05-0.34)$ & $<0.0001$ \\
\hline \multicolumn{4}{|l|}{ protein/creatinine } \\
\hline \multicolumn{4}{|l|}{ ratio $(\mathrm{g} / \mathrm{mg})$} \\
\hline Hemoglobin (mg/dL) & $13.5(13.3-14.3)$ & $14(12.6-14.7)$ & 0.70 \\
\hline Neutrophil $\left(\mathrm{mm}^{3}\right)$ & $3100(2520-3515)$ & $4260(3287-5937)$ & $<0.0001$ \\
\hline Lymphocyte $\left(\mathrm{mm}^{3}\right)$ & $2190(1690-2583)$ & $2100(1582-2710)$ & 0.29 \\
\hline
\end{tabular}

BMI body mass index, SBP systolic blood pressure, DBP diastolic blood pressure, ALT alanine aminotransferase, AST aspartate aminotransferase, LDL low-density lipoprotein, HDL high-density lipoprotein, eGFR estimated glomerular filtration rate.

difference in terms of sTWEAK between healthy subects and eGFR group 2 and group 3 patients $(\mathrm{p}=0.02$ and $\mathrm{p}=0.03$, respectively).

\section{Predictors of CIMT in Rtx patients}

To investigate the relationship between atherosclerosis and inflammation, we performed a univariate correlation analysis. CIMT was positively correlated with STWEAK and NLR in Rtx patients $(\mathrm{r}=0.81, \mathrm{p}<0.0001$ and $\mathrm{r}=0.33$, $\mathrm{p}=0.006$, respectively). Serum sTWEAK was also positively correlated with NLR ( $\mathrm{r}=0.37, \mathrm{p}=0.002)$.

We also checked the relation between serum uric acid and inflammatory markers. There were no relationship between serum uric acid and inflammatory markers including hs-CRP and NLR ( $\mathrm{r}=0.01, \mathrm{p}=0.89$ and $\mathrm{r}=0.05$, $\mathrm{p}=0.66$, respectively).

Table 2 sTWEAK, hs-CRP, NLR and CIMT values of the healthy subjects and renal transplant patients

\begin{tabular}{llllll}
\hline Parameters & $\begin{array}{l}\text { Healthy subjects Median } \\
\text { Median }(\mathrm{QR})(\mathrm{n}=25)\end{array}$ & $\begin{array}{l}\text { Renal transplant patients } \\
(\mathrm{n}=70) \text { Median }(\mathrm{QQR})\end{array}$ & $\mathrm{P}$ & $\rho(\mathrm{P})^{\mathrm{a}}$ & $\rho(\mathrm{P})^{\mathrm{b}}$ \\
\hline hs-CRP $(\mathrm{mg} / \mathrm{L})^{*}$ & $3,3(2.25-5.8)$ & $8.9(4.97-14)$ & $<0.0001$ & $0,15(\mathrm{p}=0.25)$ & $0.09(\mathrm{p}=0.46)$ \\
STWEAK $(\mathrm{pg} / \mathrm{mL})^{*}$ & $457(320.5-537.8)$ & $388.9(324.5-426.9)$ & 0.01 & - & $0.81(\mathrm{p}<0.0001)$ \\
NLR & $1.16(0.97-1.5)$ & $2.18(1.4-2.8)$ & 0.001 & $0.37(\mathrm{p}=0.002)$ & $0.33(\mathrm{p}=0.006)$ \\
CIMT $(\mathrm{mm})$ & $0.4(0.4-0.5)$ & $0.5(0.4-0.9)$ & $<0.0001$ & $0.81(\mathrm{p}<0.0001)$ & - \\
\hline
\end{tabular}

sTWEAK soluble TNF-like weak inducer of apoptosis, NLR neutrophil-to-lymphocyte ratio, CIMT carotid intima media thickness, hs-CRP high sensitive C-reactive protein, IQR interquartile range, * represents the variables in median (IQR).

a Spearman rank univariate analysis for STWEAK in renal transplant patients.

b Spearman rank univariate analysis for CIMT in renal transplant patients. 
Table 3 sTWEAK, NLR, CIMT results according to eGFR groups of renal transplant patients and healthy subjects

\begin{tabular}{|c|c|c|c|c|c|}
\hline Parameters & $\begin{array}{l}\text { Healthy subjects }(n=25) \\
\text { Median (IQR) }\end{array}$ & $\begin{array}{l}\text { eGFR group } 1(n=23) \\
\text { Median (IQR) }\end{array}$ & $\begin{array}{l}\text { eGFR group } 2(n=24) \\
\text { Median (IQR) }\end{array}$ & $\begin{array}{l}\text { eGFR group } 3(n=23) \\
\text { Median (IQR) }\end{array}$ & $\mathrm{p}^{*}$ \\
\hline MDRD (mL/min) & $107(52.7)$ & $83(13.56)$ & $63(8.7)$ & $42(22)$ & $<0.0001$ \\
\hline hs-CRP (mg/L) & $3.3(2.25-5.8)$ & $8.7(5.6-12.6)$ & $9.45(4.92-14)$ & $9.0(3.3-18)$ & $<0.0001$ \\
\hline NLR & $1.16(0.54)$ & $2.25(1)$ & $2.16(1.5)$ & $2.58(2.5)$ & 0.005 \\
\hline CIMT (mm) & $0.4(0.1)$ & $0.7(0.4)$ & $0.6(0.4)$ & $0.7(0.5)$ & $<0.001$ \\
\hline sTWEAK (pg/mL) & $457(320.5-537.8)$ & 399.5 (329-439) & $382.9(308.6-421.5)$ & $361.2(323.4-426.5)$ & 0.04 \\
\hline
\end{tabular}

sTWEAK soluble TNF-like weak inducer of apoptosis, NLR neutrophil-to-lymphocyte ratio, CIMT carotid intima-media thickness, MDRD modification of diet in renal disease, hs-CRP high sensitive C-reactive protein, IQR interquartile range, eGFR estimated glomerular filtration rate, *p value between all groups.

In the multivariate analysis, only sTWEAK was found to be an independent variable of increased CIMT. Regression results are shown in Table 4.

\section{Discussion}

The major findings of the present study were as follows; i) STWEAK levels were significantly decreased and CIMT, NLR and hs-CRP were significantly increased in Rtx patients compared to healthy subjects, ii) sTWEAK was positively correlated with CIMT and NLR but not with hs-CRP in Rtx patients, iii) sTWEAK was found to be an independent predictor of increased CIMT in Rtx patients.

In recent years, sTWEAK was discovered as a multifunctional cytokine that was related to the TNF super family. Description of TWEAK in kidney was reported for the first time by Justo et al. [25] in a mouse model of folate-induced acute kidney injury. In the following years,

\begin{tabular}{|c|c|c|c|}
\hline Parameters & Standardized Beta $^{\neq}$ & $P$ value & $95 \% \mathrm{Cl}$ \\
\hline \multicolumn{4}{|l|}{ Step 1} \\
\hline Age (years) & 0.17 & 0.18 & $-0.003-0.016$ \\
\hline Phosphorus (mg/dL) & -0.03 & 0.78 & $-0.179-0.135$ \\
\hline LDL (mg/dL) & -0.11 & 0.37 & $-0.006-0.002$ \\
\hline hs-CRP (mg/L) & 0.13 & 0.30 & $-0.007-0.023$ \\
\hline NLR & 0.03 & 0.84 & $-0.089-0.109$ \\
\hline TWEAK (pg/mL) & 0.42 & 0.001 & $0.001-0.004$ \\
\hline \multicolumn{4}{|l|}{ adjusted $r^{2}=0.18$} \\
\hline \multicolumn{4}{|l|}{${ }^{*} p=0.008$} \\
\hline \multicolumn{4}{|l|}{ Step 5} \\
\hline TWEAK (pg/mL) & 0.42 & $<0.001$ & $0.001-0.004$ \\
\hline \multicolumn{4}{|l|}{ adjusted $r^{2}=0.20$} \\
\hline \multicolumn{4}{|l|}{${ }^{*} p<0.0001$} \\
\hline $\begin{array}{l}\text { *It was define to model s } \\
\text { density lipoprotein, hs-C } \\
\text { to-lymphocyte ratio, TW } \\
\text { of apoptosis. } \\
\text { ₹ Standardized Beta mear } \\
\text { the variables. }\end{array}$ & $\begin{array}{l}\text { tatistic for each step. } \mathrm{Cl} \\
\text { RP high sensitive C-reac } \\
\text { EAK tumor necrosis fac }\end{array}$ & $\begin{array}{l}\text { onfidence } \\
\text { ive protein } \\
\text { tor-like we } \\
\text { n coefficien }\end{array}$ & $\begin{array}{l}\text { terval, LDL low } \\
\text { NLR neutrophil- } \\
k \text { inducer }\end{array}$ \\
\hline
\end{tabular}

Yilmaz et al. [26] demonstrated that a decline in eGFR was accompanied by a gradual reduction in STWEAK in CKD patients. The same group also showed that endothelial dysfunction and decreased sTWEAK were independently associated with cardiovascular outcomes in predialytic CKD patients [21]. In addition, treatment of type 1 hypertensive diabetic CKD with amlodipin and/or valsartan improves endothelial dysfunction and normalizes sTWEAK [27]. During the preparation of the present manuscript, Gungor et al. [28] described a possible role for sTWEAK in the pathogenesis of atherogenesis in the RTx population.

In our cohort, Rtx patients had lower sTWEAK levels compared to healthy subjects. However, median values of sTWEAK levels of our Rtx patient were higher compared to CKD patients in the cohorts of Yilmaz et al. [21] and Carrero et al. [29] (388.97 pg/mL vs. $245.5 \mathrm{pg} / \mathrm{mL}$ and $208 \mathrm{pg} / \mathrm{mL}$, respectively). We also investigated the relation between sTWEAK and kidney function in Rtx patients. After the classification of Rtx patients according to their eGFR, we found that sTWEAK levels were significantly decreased when eGFR values were decreased. These findings are in accord with those obtained by Yilmaz et al. [26]. Therefore, we hypothesized that the decrease of sTWEAK among eGFR groups in Rtx patients might be associated with ongoing inflammation in this population.

Inflammation seen after Rtx has a complex etiology, including a role for innate and acquired immunity. Macrophages and neutrophils play an important role in the pathogenesis of inflammation and acute and chronic allograft rejection [30]. However, ongoing inflammation in Rtx patients is much less intense compared to CKD and ESRD patients. Neutrophil-to-lymphocyte ratio was introduced as a potential marker to determine inflammation in cardiac and non-cardiac disorders [10-12]. In a previous study, we demonstrated that NLR was positively correlated with TNF- $\alpha$ in ESRD patients [14]. In the present study, we found that both hs-CRP and NLR were significantly increased in Rtx patients suggesting that these patients had infra-clinic, smoldering inflammation. We also showed that NLR was positively correlated with sTWEAK in this population. This finding might represent the link between inflammation and 
sTWEAK in Rtx patients. For better understanding, we illustrated this potential pathophysiological relationship in Figure 1.

Recently, Hornum et al. investigated the effect of Rtx on arterial function and they showed a significant improvement in endothelial function, mean arterial pressure and plasma vWF levels but not in plasma CRP and albumin levels [31]. Seyahi et al. also demonstrated that renal transplantation did not reverse or halt the progression of coronary artery calcification [32]. Our results are in accord with these studies - Rtx patients had higher CIMT compared to healthy subjects. Moreover, sTWEAK and NLR were found to be significantly and positively correlated with CIMT. Since STWEAK is also related to eGFR, we hypothesize that decreased allograft function is associated with inflammation and atherosclerosis in a vicious circle. Therefore, sTWEAK is most probably a pathogenic cytokine rather than only a biomarker in Rtx patients.

The role of TWEAK in the renal tubulointerstitial inflammation was highlighted by Ortiz et al. [33] as an activator of nuclear factor kappa $\beta(\mathrm{NFk} \beta)$, which has effects on transcription of inflammatory cytokines including MCP-1, RANTES, and IL-6 [33]. Tacrolimus, widely used as an immunosuppressive agent in Rtx patients, has been shown to down-regulate NFk $\beta$ pathway and induce apoptosis of both activated $\mathrm{T}$ cells and macrophages, in IL-10 knockout mice [34]. Recently, Du et al. [35] showed the importance of suppression of NFk $\beta$ by cyclosporin $\mathrm{A}$ and tacrolimus in renal tubular cells. In our study, out of 70 Rtx patients, 60 were receiving tacrolimus as a main immunosuppressive therapy. Despite the absence of any correlation between tacrolimus usage and sTWEAK it is plausible to suggest that decreased sTWEAK levels might be a compensatory response to the inhibitory effects of tacrolimus on the NFkB pathway (Figure 1).

Our study had two main limitations. First, this was a cross-sectional analysis of Rtx patients focusing on CIMT and sTWEAK. Second, the sample size was relatively small. This was not a prospective controlled study and therefore, we cannot draw cause-and-effect relationships from our findings.

None of our renal transplant patient died after they enrolled in this study. Hence, we could not analyze the relation between inflammation markers and mortality. However, a prospective study looking at the significance of these markers in mortality should be done.

\section{Conclusions}

sTWEAK might have a role in the pathogenesis of ongoing atherosclerosis in Rtx patients. Rtx may not completely reverse non-traditional risk factors including inflammation and atherosclerosis. When considered together, ongoing inflammation and interactions between drugs and macrophages might explain why CKD and ESRD patients had lower sTWEAK levels than Rtx patients. Renal transplant patients should be assessed to their inflammatory and uremic status individually. There are many missing pieces in the puzzle and nothing is yet known about the clinical implications of sTWEAK in Rtx

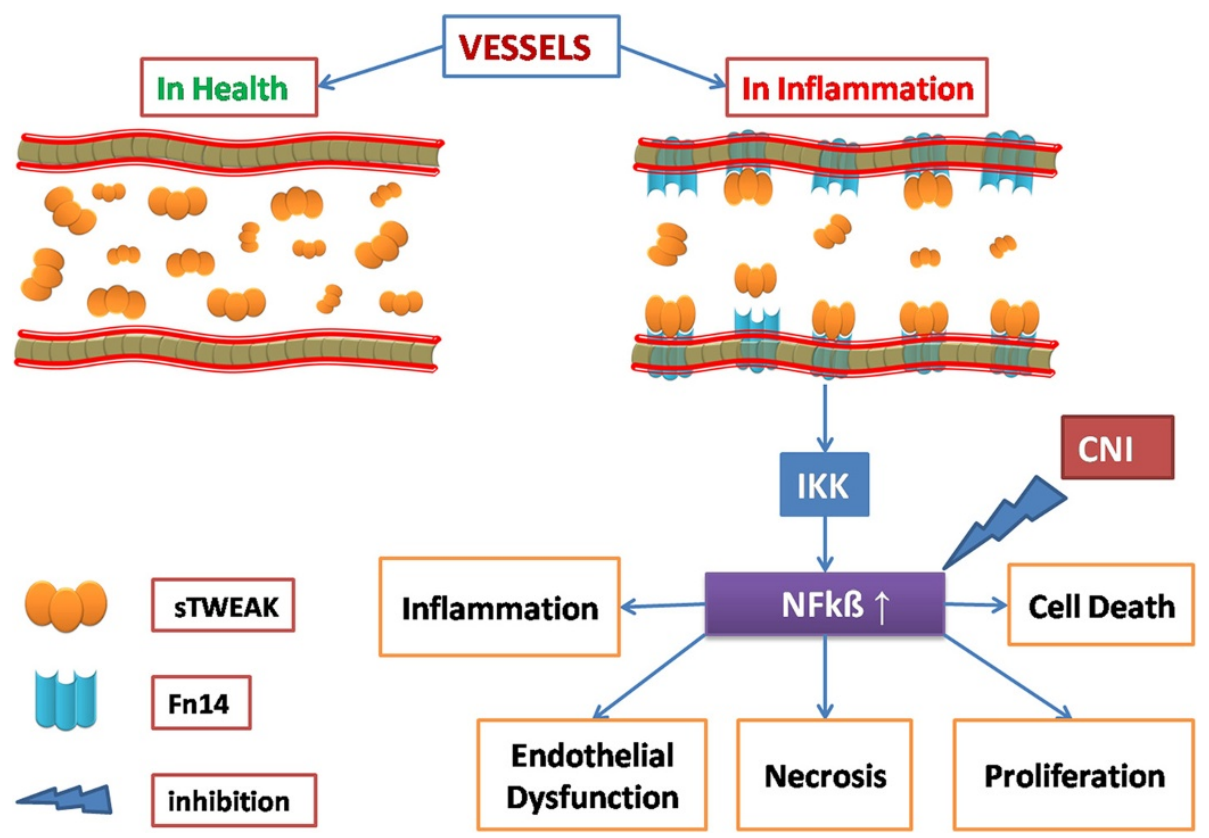

Figure 1 The relationship between STWEAK and inflammation in renal transplant patients. 
patients. Further randomized controlled trials and experimental studies investigating the exact roles of sTWEAK in the pathogenesis of atherosclerosis in renal transplantation patients are needed.

\section{Competing interests}

The authors declare that they have no conflict of interest.

\section{Authors' contributions}

KT: Participated in research design and performance of the research, data analysis, participated in the writing of the paper. HZT: Participated in research design. FME: Participated in the performance of the research. AT: Participated in laboratory analysis. ZB: Participated in the performance of the research. HO: Participated in radiological measurements. AG: Participated in data analysis. EEG: Participated in the data analysis. MK: Participated in the reseach design. YS: Participated in data analysis. OO: Participated in data analysis of radiological measurements. ST: Participated in the reseach design. AC: Participated in the editing of the manuscript. All authors read and approved the final manuscript.

\section{Acknowledgements}

Dr. A. Gaipov received grant support from the ERA-EDTA fellowship program.

\section{Author details}

'Department of Nephrology, Erzincan University Mengucek Gazi Training and Reseach Hospital, Erzincan, Turkey. ${ }^{2}$ Department of Nephrology, Necmettin Erbakan University Meram School of Medicine, Konya, Turkey. ${ }^{3}$ Department of Radiology, Necmettin Erbakan University Meram School of Medicine, Konya, Turkey. ${ }^{4}$ Department of Biochemistry, Necmettin Erbakan University Meram School of Medicine, Konya, Turkey. ${ }^{5}$ Department of Cardiology, Necmettin Erbakan University Meram School of Medicine, Konya, Turkey. ${ }^{6}$ Nephrology Clinic, Dialysis and Renal Transplant Center, 'C.I. PARHON' University Hospital, 'Gr. T. Popa' University of Medicine and Pharmacy, lasi, Romania.

Received: 8 December 2012 Accepted: 8 July 2013

Published: 12 July 2013

\section{References}

1. Collins AJ: Cardiovascular mortality in end-stage renal disease. Am J Med Sci 2003, 325(4):163-7.

2. Morris ST, McMurray JJ, Rodger RS, Farmer R, Jardine AG: Endothelial dysfunction in renal transplant recipients maintained on cyclosporine. Kidney Int 2000, 57(3):1100-6.

3. Vanholder R, Massy Z, Argiles A, Spasovski G, Verbeke F, Lameire N: Chronic kidney disease as cause of cardiovascular morbidity and mortality. Nephrol Dial Transplant 2005, 20(6):1048-56.

4. Parfrey PS, Foley RN, Harnett JD, Kent GM, Murray DC, Barre PE: Outcome and risk factors for left ventricular disorders in chronic uraemia. Nephrol Dial Transplant 1996, 11(7):1277-85.

5. Cheung AK, Sarnak MJ, Yan G, Dwyer JT, Heyka RJ, Rocco MV, et al: Atherosclerotic cardiovascular disease risks in chronic hemodialysis patients. Kidney Int 2000, 58(1):353-62.

6. Muntner P, He J, Astor BC, Folsom AR, Coresh J: Traditional and nontraditional risk factors predict coronary heart disease in chronic kidney disease: results from the atherosclerosis risk in communities study. J Am Soc Nephrol 2005, 16(2):529-38.

7. Himmelfarb J, Stenvinkel P, Ikizler TA, Hakim RM: The elephant in uremia: oxidant stress as a unifying concept of cardiovascular disease in uremia. Kidney Int 2002, 62(5):1524-38.

8. Stenvinkel P, Carrero JJ, Axelsson J, Lindholm B, Heimburger O, Massy Z: Emerging biomarkers for evaluating cardiovascular risk in the chronic kidney disease patient: how do new pieces fit into the uremic puzzle? Clin J Am Soc Nephrol 2008, 3(2):505-21.

9. Turkmen K, Gorgulu N, Uysal M, Ozkok A, Sakaci T, Unsal A, et al: Fetuin-A, inflammation, and coronary artery calcification in hemodialysis patients. Indian J Nephrol 2011, 21(2):90-4.

10. Tamhane UU, Aneja S, Montgomery D, Rogers EK, Eagle KA, Gurm HS: Association between admission neutrophil to lymphocyte ratio and outcomes in patients with acute coronary syndrome. Am J Cardiol 2008, 102(6):653-7.

11. Nunez J, Nunez E, Bodi V, Sanchis J, Minana G, Mainar L, et al: Usefulness of the neutrophil to lymphocyte ratio in predicting long-term mortality in ST segment elevation myocardial infarction. Am J Cardiol 2008, 101(6):747-52.

12. Walsh SR, Cook EJ, Goulder F, Justin TA, Keeling NJ: Neutrophil-lymphocyte ratio as a prognostic factor in colorectal cancer. J Surg Oncol 2005, 91(3):181-4

13. Duffy BK, Gurm HS, Rajagopal V, Gupta R, Ellis SG, Bhatt DL: Usefulness of an elevated neutrophil to lymphocyte ratio in predicting long-term mortality after percutaneous coronary intervention. Am J Cardiol 2006, 97(7):993-6.

14. Turkmen K, Guney I, Yerlikaya FH, Tonbul HZ: The Relationship Between Neutrophil-to-Lymphocyte Ratio and Inflammation in End-Stage Renal Disease Patients. Ren Fail 2012, 34(2):155-9.

15. Burkly LC, Michaelson JS, Zheng TS: TWEAK/Fn14 pathway: an immunological switch for shaping tissue responses. Immunol Rev 2011, 244(1):99-114.

16. Wiley SR, Winkles JA: TWEAK, a member of the TNF superfamily, is a multifunctional cytokine that binds the TweakR/Fn14 receptor. Cytokine Growth Factor Rev 2003, 14(3-4):241-9.

17. Polek TC, Talpaz M, Darnay BG, Spivak-Kroizman T: TWEAK mediates signal transduction and differentiation of RAW264.7 cells in the absence of Fn14/TweakR. Evidence for a second TWEAK receptor. J Biol Chem 2003, 278(34):32317-23.

18. Ortiz A, Sanz AB, Munoz Garcia B, Moreno JA, Sanchez Nino MD, Martin-Ventura $\mathrm{JL}$, et al: Considering TWEAK as a target for therapy in renal and vascular injury. Cytokine Growth Factor Rev 2009, 20(3):251-8.

19. Urbonaviciene G, Martin-Ventura JL, Lindholt JS, Urbonavicius S, Moreno JA, Egido J, et al: Impact of soluble TWEAK and CD163/TWEAK ratio on long-term cardiovascular mortality in patients with peripheral arterial disease. Atherosclerosis 2011, 219(2):892-9.

20. Blanco-Colio LM, Martin-Ventura JL, Munoz-Garcia B, Orbe J, Paramo JA, Michel JB, et al: Identification of soluble tumor necrosis factor-like weak inducer of apoptosis (sTWEAK) as a possible biomarker of subclinical atherosclerosis. Arterioscler Thromb Vasc Biol 2007, 27(4):916-22.

21. Yilmaz MI, Sonmez A, Ortiz A, Saglam M, Kilic S, Eyileten T, et al: Soluble TWEAK and PTX3 in nondialysis CKD patients: impact on endothelial dysfunction and cardiovascular outcomes. Clin J Am Soc Nephrol 2011, 6(4):785-92.

22. Friedewald WT, Levy RI, Fredrickson DS: Estimation of the concentration of low-density lipoprotein cholesterol in plasma, without use of the preparative ultracentrifuge. Clin Chem 1972, 18(6):499-502.

23. Levey AS, Berg RL, Gassman JJ, Hall PM, Walker WG: Creatinine filtration, secretion and excretion during progressive renal disease. Modification of Diet in Renal Disease (MDRD) Study Group. Kidney Int Supp/ 1989, 27:573-80.

24. Benedetto FA, Mallamaci F, Tripepi G, Zoccali C: Prognostic value of ultrasonographic measurement of carotid intima media thickness in dialysis patients. J Am Soc Nephrol 2001, 12(11):2458-64.

25. Justo P, Sanz AB, Sanchez-Nino MD, Winkles JA, Lorz C, Egido J, et al: Cytokine cooperation in renal tubular cell injury: the role of TWEAK. Kidney Int 2006, 70(10):1750-8.

26. Yilmaz MI, Carrero JJ, Ortiz A, Martin-Ventura JL, Sonmez A, Saglam M, et al: Soluble TWEAK plasma levels as a novel biomarker of endothelial function in patients with chronic kidney disease. Clin J Am Soc Nephrol 2009, 4(11):1716-23.

27. Yilmaz MI, Qureshi AR, Carrero JJ, Saglam M, Suliman ME, Caglar K, et al: Predictors of carotid artery intima-media thickness in chronic kidney disease and kidney transplant patients without overt cardiovascular disease. Am J Nephrol 2010, 31(3):214-21.

28. Gungor O, Kismali E, Sisman AR, Kircelli F, Carrero JJ, Tatar E, et al: The relationships between serum STWEAK, FGF-23 levels, and carotid atherosclerosis in renal transplant patients. Ren Fail 2013, 35(1):77-81.

29. Carrero JJ, Ortiz A, Qureshi AR, Martin-Ventura JL, Barany P, Heimburger O, et al: Additive effects of soluble TWEAK and inflammation on mortality in hemodialysis patients. Clin J Am Soc Nephrol 2009, 4(1):110-8.

30. Wood KJ, Goto R: Mechanisms of rejection: current perspectives. Transplantation 2012, 93(1):1-10.

31. Hornum M, Clausen P, Idorn T, Hansen JM, Mathiesen ER, Feldt-Rasmussen B: Kidney transplantation improves arterial function measured by pulse 
wave analysis and endothelium-independent dilatation in uraemic patients despite deterioration of glucose metabolism. Nephrol Dial Transplant 2011, 26(7):2370-7.

32. Seyahi N, Cebi D, Altiparmak MR, Akman C, Ataman R: Pekmezci S, et al. Nephrol Dial Transplant: Progression of coronary artery calcification in renal transplant recipients; 2011.

33. Sanz AB, Justo P, Sanchez-Nino MD, Blanco-Colio LM, Winkles JA, Kreztler M, et al: The cytokine TWEAK modulates renal tubulointerstitial inflammation. J Am Soc Nephrol 2008, 19(4):695-703.

34. Yoshino T, Nakase H, Honzawa Y, Matsumura K, Yamamoto S, Takeda Y, et al: Immunosuppressive effects of tacrolimus on macrophages ameliorate experimental colitis. Inflamm Bowel Dis 2010, 16(12):2022-33.

35. Du S, Hiramatsu N, Hayakawa K, Kasai A, Okamura M, Huang T, et al: Suppression of NF-kappaB by cyclosporin a and tacrolimus (FK506) via induction of the C/EBP family: implication for unfolded protein response. $\mathrm{J}$ Immunol 2009, 182(11):7201-11.

doi:10.1186/1471-2369-14-144

Cite this article as: Turkmen et al:: Soluble TWEAK independently predicts atherosclerosis in renal transplant patients. BMC Nephrology 2013 14:144.

\section{Submit your next manuscript to BioMed Central and take full advantage of:}

- Convenient online submission

- Thorough peer review

- No space constraints or color figure charges

- Immediate publication on acceptance

- Inclusion in PubMed, CAS, Scopus and Google Scholar

- Research which is freely available for redistribution 